\title{
Asthma and dental caries
}

\author{
Abstracted from \\ Alavaikko S, Jaakkola MS, Tjäderhane L, Jaakkola JJ. \\ Asthma and caries: a systematic review and meta-analysis. \\ Am J Epidemiol. 2011; 174: 631-641 \\ Address for correspondence Dr. Jouni J. K. Jaakkola, \\ Center for Environmental and Respiratory Health Research, Institute of Health Sciences, \\ University of Oulu, Aapistie 1, P.O. Box 5000, 90014 Oulu, Finland (e-mail: jouni.jaakkola@oulu.fi).
}

\section{Question: Is there a relationship between asthma and dental caries?}

Data sources Medline, government reports and conference proceedings were searched.

Study selection Case-control, cohort or cross sectional studies were included if they provided relevant and applicable quantitative information on the relation between asthma and caries, had an independent study population and adequate definitions of asthma and caries and appropriate measurement of caries.

Data extraction and synthesis Data were extracted independently by two reviewers and quality was assessed using the NewcastleOttawa Quality Assessment Scale. Fixed- and random-effects models were used for the analyses. Heterogeneity and publication bias were evaluated.

Results Eighteen articles were included; 11 provided information on primary dentition and 15 on permanent dentition. Random-effects models showed a significant association between asthma and caries for both primary and permanent dentition, the odds ratios being 2.73 (95\% Cl: 1.61, 4.64) and 2.04 (95\% Cl: 1.44, 2.89), respectively. Conclusions Evidence from this analysis suggests that asthma doubles the risk of caries in both primary and permanent dentition. Publication bias diagnostics and simulation suggested possible overestimation of the summary odds ratio for permanent dentition but not for primary dentition. Physicians and dentists should recommend preventive measures against caries for people with asthma.

\section{Commentary}

Caries and asthma are two of the most prevalent childhood diseases. ${ }^{1,2}$ There are both epidemiologic and clinical reasons to discover any association between them. Demonstrating an association may guide efforts to identify mechanisms of disease, underscore the importance of caries prevention and suggest intervention strategies for asthma patients both in terms of clinical practice and public health policy.

The present systematic review was carefully conducted and the analysis was rigorous. Including studies of different designs makes assigning the level of evidence difficult, but quality assessment of individual studies was thorough. The authors undertook subgroup analyses, which are useful when dissecting out the unique character of disease associations from accumulated individual studies of variable quality.
Summary estimates in separate meta-analyses showed increased caries incidence in primary and permanent dentition of asthma patients, however the questionable exclusion of a large casecontrol study ${ }^{3}$ because of suspicion 'that there were errors in the published data' casts doubt over the authors' conclusion of association between asthma and caries in permanent teeth. This decision also placed the results somewhat at odds with an earlier review by Maupomé, et al. ${ }^{4}$ The Maupomé review concluded that asthma 'may not be a risk factor for dental caries', whereas the present review and meta-analyses found that 'asthma significantly increases the risk of caries.'

As with many retrospective analyses this review has several weaknesses. Definition of asthma by physician diagnosis, asthma symptoms or asthma medications is sufficiently heterogeneous to warrant a cautious approach. Caries rates based on retrospective evaluation of dental records may not be sufficiently sensitive for great confidence in the data. Finally the authors did not report attempting to contact authors of seven excluded studies for possible clarification of missing information. Despite these weaknesses, the conclusion that there is a nearly two-fold increase in caries experience in the primary teeth of children with asthma compared to children without asthma is supported by the highest quality studies reported in this review.

There are many speculations in this and other papers about reasons for an association between asthma and caries, including increased inflammation, decreased salivary flow and lower plaque pH. ${ }^{5}$ To date high quality evidence for these or other plausible mechanisms has not been reported.

Based on this systematic review greater oral health vigilance is warranted for children with asthma.

\section{Joseph Matthews}

General dentist - Los Alamos, New Mexico, USA

1. Masoli M, Fabian D, Holt S, Beasley R; Global Initiative for Asthma (GINA) Program. The global burden of asthma: executive summary of the GINA Dissemination Committee report. Allergy. 2004; 59: 469-478

2. Petersen PE, Bourgeois D, Ogawa H, Estupian-Day S, Ndiaye C. The global burden of oral diseases and risks to oral health. Bull World Health Organ. 2005; 83: 661-669

3. Shulman JD, Taylor SE, Nunn ME. The association between asthma and dental caries in children and adolescents: a population-based case-control study. Caries Res. 2001; 35: $240-246$

4. Maupomé G, Shulman JD, Medina-Solis CE, Ladeinde O. Is there a relationship between asthma and dental caries? : a critical review of the literature. / Am Dent Assoc. 2010; 141: 1061-1074

5. Stensson M, Wendt LK, Koch G, Oldaeus G, Lingström P, Birkhed D. Caries prevalence, caries-related factors and plaque $\mathrm{pH}$ in adolescents with long-term asthma. Caries Res. 2010; 44: 540-546

Evidence-Based Dentistry (2012) 13, 41. doi:10.1038/sj.ebd.6400854 